\title{
Preoperative Neck Angulation is Associated with Aneurysm Sac Growth Due to Persistent Type la Endoleak after Endovascular Abdominal Aortic Aneurysm Repair
}

\author{
Yoshimasa Seike, MD, ${ }^{1}$ Tetsuya Fukuda, MD, PhD, ${ }^{2}$ Koki Yokawa, MD, ${ }^{1}$ Yosuke Inoue, MD, ${ }^{1}$ \\ Takayuki Shijo, MD, ${ }^{1}$ Kyokun Uehara, MD, PhD, ${ }^{1}$ Hiroaki Sasaki, MD, PhD, ${ }^{1}$ and \\ Hitoshi Matsuda, MD, PhD'
}

Objective: This study aims to determine how instructions for use affect the occurrence of aneurysm sac growth and endoleaks after an endovascular aneurysm repair (EVAR).

Materials and Methods: We reviewed 302 patients who underwent EVAR for abdominal aortic aneurysm between 2007 and 2013, and we were able to enroll 159 patients (74\% men, mean age $78 \pm 7$ years) with adequate data (mean follow-up; $48 \pm 20$ months).

Results: The angle of the proximal landing zone (LZ) (hazard ratio: 1.02, 95\% confidence interval: 1.00-1.03, $\mathrm{p}=0.01$ ) was recognized as an independent risk factor of sac growth ( $\geq 5 \mathrm{~mm})$. The receiver operating characteristics curve (area under the curve: 0.72) showed a cutoff value of $47^{\circ}$ of the minimum angle of the proximal $L Z$ to predict sac growth. Freedom rates for persistent type la endoleaks were also found to be lower in the angulated group than those in the other groups ( $p=0.0095$, log-rank).

Conclusion: The angle of the proximal LZ was identified as an independent risk factor for sac growth post-EVAR. The incidence of persistent type la endoleaks was significantly higher in the angulated group.

\footnotetext{
${ }^{1}$ Department of Cardiovascular Surgery, National Cerebral and Cardiovascular Center, Suita, Osaka, Japan

${ }^{2}$ Department of Radiology, National Cerebral and Cardiovascular Center, Suita, Osaka, Japan
}

Received: April 13, 2020; Accepted: May 20, 2020

Corresponding author: Hitoshi Matsuda, MD. Department of Cardiovascular Surgery, National Cerebral and Cardiovascular Center, 6-1 Kishibe-shimmachi, Suita, Osaka 564-8565, Japan

Tel: +81-6-6170-1070, Fax: +81-6-6170-1424

E-mail: hitmat@mist.ocn.ne.jp

(cc) BY-NC-SA (92020 The Editorial Committee of Annals of Vascular Diseases. This article is distributed under the terms of the Creative Commons Attribution License, which permits use, distribution, and reproduction in any medium, provided the credit of the original work, a link to the license, and indication of any change are properly given, and the original work is not used for commercial purposes. Remixed or transformed contributions must be distributed under the same license as the original.
Keywords: endovascular aneurysm repair, instructions for use, angulation, endoleak

\section{Introduction}

Endovascular aneurysm repair (EVAR) has been increasingly used for infrarenal abdominal aortic aneurysm (AAA) repair, mainly due to its lower short-term morbidity and mortality compared with open surgery in the elderly. Nonetheless, a major disadvantage of this technique is the possibility for secondary intervention due to any type of endoleaks. ${ }^{1-3)}$

Recently, various stent grafts have become commercially available, and each device has its own instructions for use (IFU), which indicate the anatomic restrictions for the device selection to minimize adverse outcomes after EVAR. These anatomic restrictions mainly consist of the aortic neck diameter, aortic neck length, aortic neck angulation, and the parameters of the iliac arteries. However, many elderly patients necessitate and undergo EVAR even if the anatomy of their AAA is beyond the IFU parameters. Besides its clinical importance, IFU adherence remains controversial as that is said by the reported studies. ${ }^{4-6)}$

This study aimed to determine how IFU influences the incidence of sac growth and endoleaks post-EVAR and to recognize the computed tomography (CT) features of IFU, which may predict adverse events post-EVAR.

\section{Materials and Methods}

\section{Ethics statement}

This observational study was approved by the Institutional Review Board (M30-036) at our center, and individual oral and written informed consents were waived because of its retrospective nature. 


\section{Study population}

The data were collected from hospital admission and outpatient medical records. All patients have been followed up as outpatients either at our center or by a local physician. The medical records of 302 patients who underwent EVAR between January 2007 and December 2013 were retrospectively reviewed. More contemporary cases were not studied, because we have performed intraoperative aneurysm neck and sac embolization with N-butyl2-cyanoacrylate (NBCA) to treat type Ia endoleaks during EVAR since 2014, besides using several other techniques including inferior mesenteric artery (IMA) embolization using coil devices. The patients who received CT scans for follow-up medically over 2 years after EVAR and had undergone elective EVAR for infrarenal AAA were included. Forty patients $(14.1 \%)$ with a solitary iliac aneurysm and two patients $(0.7 \%)$ with a ruptured AAA were excluded from the study. In addition, 16 patients (5.6\%) with IMA embolization and 29 patients $(1.0 \%)$ who had received warfarin therapy were also excluded from the study; as what we had previously reported, warfarin therapy was considered a substantial risk for sac enlargement after EVAR. ${ }^{7)}$ The patients with type I and type II endoleaks were included, while patients with type III and type IV endoleaks were excluded from the study. Finally, followup data were available in 159 patients (74\% men, mean age $78 \pm 7$ years, mean follow-up time $48 \pm 20$ months) (Fig. 1).

\section{Device for EVAR}

Stent graft selection depends on factors such as its availability, anatomical suitability, and the surgeon's preference. EVAR was done using Excluder (W. L. Gore and Associates, Flagstaff, AZ, USA) in 69 patients (43.4\%), Zenith (COOK Medical, Bloomington, IN, USA) in 44 $(27.7 \%)$, Endurant (Medtronic Cardiovascular, Santa Rosa, CA, USA) in 28 (17.6\%), Powerlink (Endologix, Irvine, CA, USA) in 17 (10.7\%), and Talent Abdominal (Medtronic AVE, Santa Rosa, CA, USA) in 1 (0.6\%).

\section{Measurement of CT scans}

Axial CT images with a slice thickness of $2 \mathrm{~mm}$ were used to measure the CT scan findings. Data were transferred to a three-dimensional (3D) workstation (Ziostation2 version 2.9.2.0a; Ziosoft, Inc., Tokyo, Japan), and the size of the aneurysm sac was measured using the major and minor axes. Aneurysm morphology including suprarenal angulation, neck diameter, neck length, neck angle, and maximum sac diameter was measured by the first author and one radiologist. Reverse-tapered shape was defined as the size discrepancy more than $3 \mathrm{~mm}$ within $15 \mathrm{~mm}$ below the lower renal artery orifice.

Neck angle has been defined as the angle between the

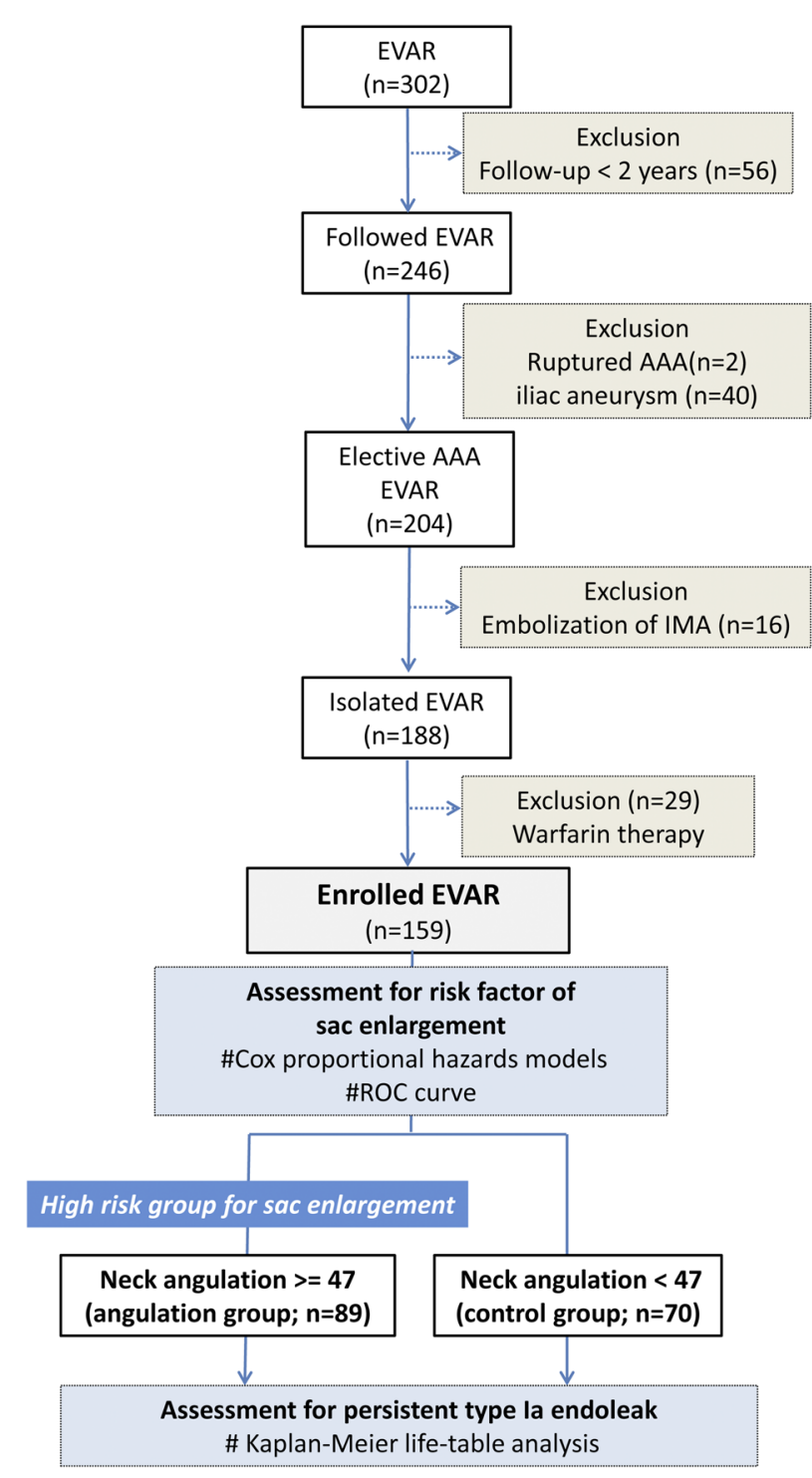

Fig. 1 Flowchart of study population and method.

axes of aneurysm and the neck of aneurysm. Figure 2 indicates the measurement of the infrarenal neck angle is the same as the neck angle. Suprarenal neck angle was defined as the angle between the axes of the suprarenal aorta and the neck of aneurysm.

During the follow-up periods, a change on the minor axis over $5 \mathrm{~mm}$ was considered significant for sac growth post-EVAR. ${ }^{8)}$ The CT scans with intravenous contrast were performed in all patients at discharge and at least twice over 2 years post-EVAR. If aneurysm sac growth was detected on CT scans postoperatively, CT angiography was performed to identify the sources of endoleaks. Persistent endoleak was defined as the endoleak which did not disappear 1 year post-EVAR, in addition to a newly developed endoleak during the follow-up period. ${ }^{7)}$ 


\section{Risk factors for sac enlargement}

Preoperative CT findings associated with IFU are presented in Table 1 and were evaluated using univariate analysis to find the risk factors for aneurysm sac growth post-EVAR. The receiver operating characteristics (ROC) curve was performed to evaluate the relation between the high-risk group for sac enlargement and preoperative CT findings including the angle of the proximal LZ and diameter of the proximal LZ on the occurrence of sac enlargement (Fig. 2).

\section{Endpoint}

To identify how IFU affects the occurrence of sac growth

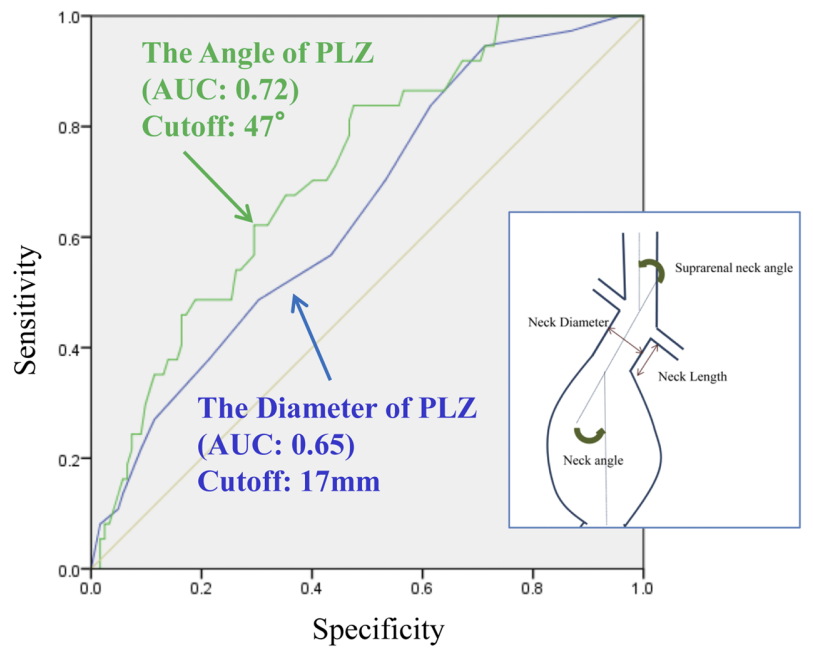

Fig. 2 Receiver operating characteristics curve analysis for angle and diameter of proximal landing zone. more than 2 years post-EVAR, risk factors related to the preoperative CT findings were examined. The patients were then divided into two groups by the cutoff value of the proximal neck angulation of $47^{\circ}$, and freedom from type Ia endoleak was compared with the secondary outcome indicated above (Fig. 1).

\section{Statistical analysis}

All statistical analyses were performed using the SPSS software (SPSS Inc., Chicago, IL, USA). Categorical data were compared using Fisher's exact test, while the continuous variables were expressed as mean \pm standard deviation and compared using a Student's t-test. A P-value of $<0.05$ was considered statistically significant for all tests. Univariate and multivariate analyses were performed using the Cox hazards regression models for evaluating the time-to-event effects of the devices for EVAR and the preoperative CT findings related to IFU, including the aneurysm size, the length of the proximal LZ, the diameter of the proximal LZ, the angle of the proximal LZ, calcification, terminal aorta diameter, the length of the distal LZ, the diameter of the distal LZ, access route diameter, and embolization of the internal iliac artery. In addition, univariate analysis was performed to evaluate the effects of both immediate type Ia and type II endoleaks on sac enlargement and persistent type Ia endoleak during the follow-up period. Clinically relevant variables with a p-value $<0.05$ in the univariate analysis were included in the multivariate regression analyses as candidates for backward stepwise variable selections.

The probabilities predicted by ROC curve of the model were plotted, and the area under the curve (AUC) was

Table 1 Preoperative computed tomography findings related to instruction for use and Cox regression analysis: predictors of sac enlargement $(>5 \mathrm{~mm})$

\begin{tabular}{|c|c|c|c|c|c|c|c|c|}
\hline \multirow{2}{*}{\multicolumn{2}{|c|}{ Covariate }} & \multirow{2}{*}{ Overall } & $\mathrm{HR}$ & $95 \% \mathrm{Cl}$ & $p$-value & $\mathrm{HR}$ & $95 \% \mathrm{Cl}$ & $p$-value \\
\hline & & & \multicolumn{3}{|c|}{ Univariate } & \multicolumn{3}{|c|}{ Multivariate } \\
\hline AAA size & $(\mathrm{mm})$ & $48 \pm 6$ & 1.07 & $1.01-1.13$ & 0.020 & 1.06 & $0.99-1.22$ & 0.057 \\
\hline Suprarenal angulation & Angle (degree) & $29 \pm 23$ & 1.01 & $1.00-1.03$ & 0.057 & & & \\
\hline \multirow[t]{7}{*}{ Proximal LZ } & Length (mm) & $32 \pm 12$ & 0.98 & $0.95-1.01$ & 0.088 & & & \\
\hline & Diameter (mm) & $19 \pm 3$ & 1.14 & $1.04-1.26$ & 0.008 & 1.11 & $1.00-1.24$ & 0.042 \\
\hline & Angle (degree) & $50 \pm 25$ & 1.02 & $1.01-1.04$ & 0.001 & 1.02 & $1.00-1.03$ & 0.010 \\
\hline & Calcification (n, \%) & $26 / 159(16 \%)$ & 1.24 & $0.48-3.19$ & 0.66 & & & \\
\hline & Mural thrombus (n, \%) & $5 / 159(3.1 \%)$ & 2.58 & $0.35-19.4$ & 0.36 & & & \\
\hline & Reverse tapered (n, \%) & $8 / 159(5.0 \%)$ & 2.77 & $0.96-7.92$ & 0.057 & & & \\
\hline & Conical (n, \%) & $3 / 159(1.9 \%)$ & 0.05 & $0.00-8.93$ & 0.54 & & & \\
\hline Terminal aorta & Diameter (mm) & $23 \pm 6$ & 1.01 & $0.96-1.06$ & 0.84 & & & \\
\hline \multirow[t]{2}{*}{ Distal LZ } & Length (mm) & $35 \pm 12$ & 1.01 & $0.98-1.03$ & 0.82 & & & \\
\hline & Diameter (mm) & $15 \pm 5$ & 1.04 & $0.98-1.11$ & 0.21 & & & \\
\hline Access route & Diameter (mm) & $7.6 \pm 1.1$ & 1.50 & $1.10-2.03$ & 0.01 & 1.32 & $0.95-1.83$ & 0.100 \\
\hline Embolization of bilateral IIAs & $(n, \%)$ & 0/159 (0\%) & - & - & - & - & - & - \\
\hline
\end{tabular}

AAA: abdominal aortic aneurysm; Cl: confidence interval; HR: hazard ratio; IIA: internal iliac artery; LZ: landing zone 
utilized to assess the differentiation of the angle and the diameter of the proximal LZ with sac enlargement $(\geq 5 \mathrm{~mm})$. Kaplan-Meier survival curves were built to evaluate persistent type Ia endoleak, and the log-rank test was applied to compare between the subgroups (bottom row of Fig. 1).

\section{Results}

\section{Operative results}

To control an intraoperative type Ia endoleak, the adjunctive procedures were performed in ten patients using a proximal aortic cuff placement (W. L. Gore and Associates, Flagstaff, AZ, USA) and in two using a Palmaz XL stent (Cordis, Miami Lakes, FL, USA). The total number of intraoperative adjunctive procedures was $12(7.5 \%)$. Residual intraoperative type Ia endoleak was detected in eight $(5.0 \%)$ patients with minor grade. Like other endoleaks, intraoperative completion angiogram showed 45 endoleaks in 53 patients (33\%), of these 44 are type II, two type III, and seven type IV.

\section{Sac growth}

Sac growth $(\geq 5 \mathrm{~mm})$ post-EVAR was identified in 37 patients $(23 \%)$. Using the Cox regression analysis, the diameter of the proximal LZ (hazard ratio [HR]: 1.11, $95 \%$ confidence interval $[\mathrm{CI}]: 1.00-1.24, \mathrm{p}=0.042)$ and angle of the proximal LZ (HR: $1.02,95 \%$ CI: $1.00-1.03$, $\mathrm{p}=0.010)$ were identified as an independent risk factor of sac growth post-EVAR (Table 1). The length of the proximal LZ was also not identified as a predictor of sac growth (HR: 0.98, 95\% CI: 0.95-1.01, p=0.088). As with other variables, no major devices were also identified as a predictor of sac growth (Excluder, HR: 1.30, $\mathrm{p}=0.43$; Zenith, HR: 0.80, $\mathrm{p}=0.55$; Endurant, HR: 0.67, $\mathrm{p}=0.41$; Powerlink, HR: 0.22, $\mathrm{p}=0.14$ ).

\section{ROC curves for the angle and diameter of the proximal landing zone}

The ROC curve for the angle of the proximal LZ showed that AUC for the predicted probabilities was $0.72(95 \%$ CI: $0.63-0.81)$. At a cutoff value of $47^{\circ}$, the sensitivity of the minimum angle of the proximal LZ for predicting sac growth was $83 \%$ with $51 \%$ specificity (Fig. 2). The

Table 2 Patient characteristics and clinical features

\begin{tabular}{|c|c|c|c|c|}
\hline \multicolumn{2}{|l|}{ Variable } & \multirow{2}{*}{$\begin{array}{c}\text { Control group Angle }<47(n=70) \\
76 \pm 8\end{array}$} & \multirow{2}{*}{$\begin{array}{c}\text { Angulated group Angle }>47(n=89) \\
79 \pm 5\end{array}$} & \multirow{2}{*}{$\begin{array}{r}\mathrm{p} \text {-value } \\
0.019\end{array}$} \\
\hline Age & (years) & & & \\
\hline Female & $\mathrm{n}(\%)$ & $11(16 \%)$ & $30(34 \%)$ & 0.010 \\
\hline Aneurysm size & $(\mathrm{mm})$ & $47 \pm 6$ & $49 \pm 6$ & 0.002 \\
\hline \multicolumn{5}{|l|}{ IFU-related features } \\
\hline Suprarenal angulation & Angle (degree) & $16 \pm 18$ & $37 \pm 25$ & $<0.001$ \\
\hline \multirow[t]{6}{*}{ Proximal landing zone } & Length (mm) & $35 \pm 11$ & $30 \pm 12$ & 0.020 \\
\hline & Diameter (mm) & $19 \pm 3$ & $20 \pm 3$ & 0.320 \\
\hline & Calcification (n, \%) & $11(16 \%)$ & $15(17 \%)$ & 1.000 \\
\hline & Mural thrombus (n, \%) & $2(2.9 \%)$ & $3(3.4 \%)$ & 0.809 \\
\hline & Reverse taper (n, \%) & $3(4.3 \%)$ & $7(7.9 \%)$ & 0.675 \\
\hline & Taper (n, \%) & $1(1.4 \%)$ & $2(2.2 \%)$ & 0.316 \\
\hline Terminal aorta & Diameter (mm) & $21 \pm 6$ & $24 \pm 7$ & 0.022 \\
\hline \multirow[t]{2}{*}{ Distal landing zone } & Length (mm) & $34 \pm 11$ & $36 \pm 13$ & 0.480 \\
\hline & Diameter (mm) & $14 \pm 4$ & $16 \pm 6$ & 0.060 \\
\hline Access route & Diameter (mm) & $7.4 \pm 1.0$ & $7.6 \pm 1.2$ & 0.300 \\
\hline \multicolumn{5}{|l|}{ Device-related features } \\
\hline Device body diameter & $(\mathrm{mm})$ & $26 \pm 3.0$ & $26 \pm 3.0$ & 0.990 \\
\hline Zenith & $(n, \%)$ & $19(27 \%)$ & $25(28 \%)$ & 0.890 \\
\hline Excluder & $(n, \%)$ & $23(33 \%)$ & $46(52 \%)$ & 0.017 \\
\hline Powerlink & $(n, \%)$ & $13(19 \%)$ & $4(4.5 \%)$ & 0.004 \\
\hline Talent AAA & $(n, \%)$ & 0 & $1(1.1 \%)$ & 0.370 \\
\hline ENDURANT & $(n, \%)$ & $15(21 \%)$ & $13(15 \%)$ & 0.260 \\
\hline \multicolumn{5}{|l|}{ Intraoperative features } \\
\hline Intraoperative blood transfusion & $(n, \%)$ & $7(10 \%)$ & $18(20 \%)$ & 0.085 \\
\hline \multicolumn{5}{|l|}{ Endoleak at hospital discharge } \\
\hline Immediate type la endoleak & $(n, \%)$ & $0(0 \%)$ & $3(3.4 \%)$ & 0.260 \\
\hline Immediate type II endoleak & $(n, \%)$ & $12(17 \%)$ & $30(34 \%)$ & 0.020 \\
\hline
\end{tabular}

AAA: abdominal aortic aneurysm; IFU: instructions for use 
ROC curve for the diameter of the proximal LZ showed the AUC of 0.65 (95\% CI: 0.48-0.69), having a cutoff number of $17 \mathrm{~mm}$. Pursuant to this result, the angle of the proximal $\mathrm{LZ}$ was detected as a significant predictor for sac growth but not its diameter.

\section{Presence of endoleak during the follow-up period}

Postoperative CT at 1 week showed 45 endoleaks in 44 patients: 3 type Ia and 30 type II endoleaks in the angulated group and 12 type II endoleaks in the control group. Immediate type II endoleaks were more frequent in the angulated group $(\mathrm{p}=0.020)$ (Table 2$)$. Using the univariate analysis, both immediate type Ia (HR: 3.89, 95\% CI: 0.52-29.3, $\mathrm{p}=0.19$ ) and type II (HR: 1.68, 95\% CI: $0.87-3.26, p=0.12$ ) endoleaks were found to be not a positive predictor of sac growth $(\geq 5 \mathrm{~mm})$ post-EVAR.

Two years after EVAR, 42 postoperative persistent endoleaks were detected, including eight type Ia, two type Ib, and 22 type II endoleaks in the angulation group $(\mathrm{n}=32)$ and one type Ib endoleak and nine type II endoleaks in the control group $(n=10)$. In eight patients who were identified with complicated persistent type Ia endoleaks, the median proximal neck angle was determined at $73^{\circ}$ (range, 50-116). The devices used were Excluder in five patients, Zenith in two, and Talent Abdominal in one. Median onset of endoleak confirmed by CT was determined to be at 18 months (range, 1-48). To treat sac enlargement in these patients, graft replacement was performed in two patients at 36 and 48 months post-EVAR, re-EVAR using a Gore Excluder aortic cuff (W. L. Gore and Associates, Flagstaff, AZ, USA) in two, and reinforcement with Palmaz stent (Cordis, Miami Lakes, FL, USA) in two.

\section{Relation between the neck angulation and persis- tent type la endoleak}

As per the result of the previous ROC curve analysis, to evaluate the relation between neck angulation and persistent type Ia endoleak, the entire cohort was divided into two groups depending on the angle of the proximal LZ: 89 patients with $47^{\circ}$ or more of the angle of the proximal LZ (angulated group, mean age $79 \pm 5$ years) and $70 \mathrm{pa}-$ tients with $47^{\circ}$ of the angle of the proximal LZ (control group, mean age $76 \pm 8$ years) (bottom row of Fig. 1).

The angulated group was determined to be older $(p=0.019)$, and they were mostly female compared with the control group ( $34 \%$ vs. $16 \%$, respectively; $p=0.043$ ). In the angulated group, the length of the proximal LZ $(35 \pm 11 \mathrm{~mm}$ vs. $30 \pm 12 \mathrm{~mm}, \mathrm{p}=0.020)$ was determined to be shorter, while the diameter of the terminal aorta $(21 \pm 6 \mathrm{~mm}$ vs. $24 \pm 7 \mathrm{~mm}, \mathrm{p}=0.022)$ was larger. Other variables revealed no differences between the two groups (Table 2). Point of difference was detected in terms of the type of endograft used, including the Excluder device and

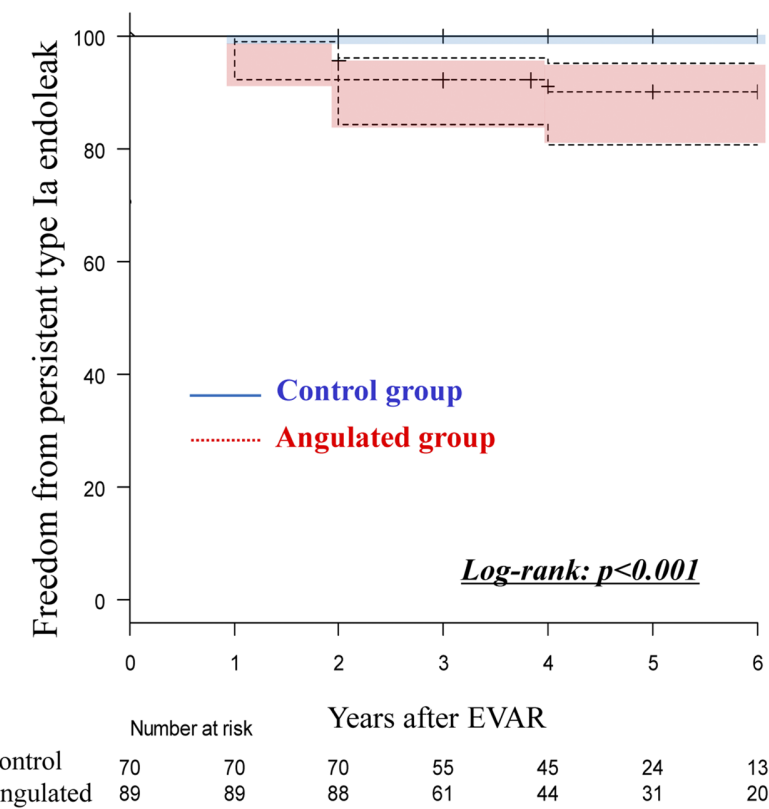

Fig. 3 The probability of freedom from persistent type la endoleak.

the Powerlink device.

Freedom from persistent type Ia endoleak post-EVAR was determined to be higher in the angulated group compared with the control group $(\mathrm{p}=0.0095, \log$-rank) (Fig. 3). Using the Cox regression analysis, the angle of proximal LZ (HR: 1.04, 95\% CI: 1.01-1.07, $\mathrm{p}=0.012$ ) and reverse-tapered shape (HR: 6.62, 95\% CI: 1.21-32.4, $\mathrm{p}=0.029)$ were identified as independent risk factors of persistent type Ia endoleak post-EVAR. The length of the proximal LZ was not identified as well as a predictor of persistent type Ia endoleak (HR: 0.95, 95\% CI: 0.89 $1.02, \mathrm{p}=0.18$ ) (Table S1). In other variables, no major devices were identified as a predictor of persistent type Ia endoleak (Excluder, HR: 2.11, $\mathrm{p}=0.31$; Zenith, HR: 0.75, $\mathrm{p}=0.73$; Endurant, HR: 0.04, $\mathrm{p}=0.48$; Powerlink, HR: $0.04, \mathrm{p}=0.51$ ).

\section{Subgroup analysis of the angulated cases between within the IFU and outside the IFU}

The angulated group ( 89 patients, $56 \%$ ) was subsequently divided into two groups in terms of the angle of the IFU $\left(60^{\circ}\right)$ as the subgroup analysis. Thirty-six patients $(36 / 89$, $40 \%$ of angulated group) were assigned to the angulation within the IFU group $\left(47^{\circ} \leq\right.$ proximal neck angle $\left.<60^{\circ}\right)$, and the other 53 patients $(53 / 89,60 \%$ of angulated group) were assigned to the angulation outside the IFU group (proximal neck angle $\geq 60^{\circ}$ ). Subgroups showed similar neck length $(31 \pm 12 \mathrm{~mm}$ in the angulation within the IFU group vs. $30 \pm 12 \mathrm{~mm}$ in the angulation outside the IFU group, $\mathrm{p}=0.51)$ and neck diameter $(20 \pm 3.3 \mathrm{~mm}$ in the angulation within the IFU group vs. $19 \pm 3.0 \mathrm{~mm}$ in the angulation outside the IFU group, $p=0.60$ ). Sac 
growth ( $\geq 5 \mathrm{~mm}$ ) post-EVAR was observed in 11 patients $(31 \%)$ of the angulation within the IFU group and in 20 patients $(38 \%)$ of the angulation outside the IFU group. Meanwhile, persistent type Ia endoleak post-EVAR was observed in five patients $(14 \%)$ of the angulation within the IFU group and in 20 patients $(9.4 \%)$ of the angulation outside the IFU group. Freedom from sac growth $(\geq 5 \mathrm{~mm})$ was similar between the two groups $(p=0.750$, log-rank).

\section{Discussion}

In 2007, a EUROSTAR study demonstrated that severe infrarenal aortic neck angulation was associated with type Ia endoleak (odds ratio 2.32, 95\% CI: 1.60-3.37, $\mathrm{p}<0.0001)$ as the risk of the AAA outside the IFU $^{9}$ was related to the need for secondary interventions (HR: 1.29, $95 \%$ CI: $1.00-1.67, p=0.049)$. However, recently, it has been reported that there is no difference in the outcomes of EVAR between the patients within and outside of the IFU. $4-6,10,11)$

Beckerman et al. concluded that there was no difference in the all-cause mortality or the aneurysm-related mortality despite most EVAR patients being treated outside the IFU.6) Moreover, aneurysm sac enlargement was identified in $11.7 \%$ of overall patients, but no significant difference between patients treated within and outside the IFU $\left.(p=0.870) .{ }^{6}\right)$ However, the detailed impact of each factor of IFU was not evaluated in their study. Likewise, Walker et al. reported that overall mortality and aneurysm-related mortality were not affected by IFU adherence in the longterm follow-up. Their Cox proportional hazard model showed that the IFU nonadherence was not predictive for all-cause mortality (HR: 1.0, $\mathrm{p}=0.910) .{ }^{10)}$

In contrast, several recent studies have focused on the proximal neck angulation associated with adverse events. ${ }^{4,12-15)}$ AbuRahma et al. have revealed its association with perioperative complications, including early type Ia endoleak and reintervention. ${ }^{13)}$ Oliveira et al. reported that severely angulated necks contributed to a higher rate of type Ia endoleaks, and these were significantly associated with freedom from neck-related secondary interventions $(86 \%$ in the angulated neck group vs. $98 \%$ in the control group, $p=0.016)$ in the long term. They also highlighted that cautious enduring follow-up is indispensable in patients treated outside the IFU. ${ }^{16)}$

These opposing perspectives led us to evaluate the threshold of the angulated neck. For this evaluation, we selected the aneurysm sac enlargement evaluated by CT (in the clinical setting), as the indication for reintervention might not be indicated purely for significant sac enlargement and be considered with the patient's background and comorbidities.
Multivariate analysis examining the anatomical features on CT scan related to IFU revealed that the angle of the proximal LZ (HR: 1.02, $\mathrm{p}=0.010$ ) and diameter of the proximal LZ (HR: 1.11, $\mathrm{p}=0.042$ ) were associated with late sac enlargement. Analysis of the angle of the proximal LZ with the ROC analysis showed that setting the cutoff value of the proximal neck angle at $47^{\circ}$ indicated a sensitivity of $83 \%$ and a specificity of $51 \%$ to predict sac growth. A proximal neck angle of $47^{\circ}$ was the threshold for sac enlargement after EVAR.

As the predictors for sac enlargement, several studies have reported the neck angle $>60^{\circ}$ was only predictable, but other degrees have yet to be investigated. ${ }^{12-17)}$ The subgroup comparison in our study, limited to the angulated group, was conducted for the proximal neck angle of $60^{\circ}$, the angle for the IFU. Interestingly, the rates of freedom from sac enlargement indicated no difference between the two groups, and proximal neck angle $>47^{\circ}$ was again identified as the threshold for developing late sac enlargement even if the AAA was within the IFU.

Among all types of endoleaks after the EVAR, type Ia endoleak was recognized as the failure of EVAR and directly related to sac enlargement. Previous studies have reported the incidences of persistent type Ia endoleaks, ranging from $2.5 \%$ to $11.4 \%$, and type Ia endoleaks have reportedly occurred in several stent graft systems. ${ }^{12-17)}$

Regarding the mechanism underlying the persistent type Ia endoleak due to neck angulation, Rahmani et al. reported that proximal angulation decreases the necessary pull-down force and results in dislodging of the endograft in bovine aorta. This in vitro study showed that the pulldown forces decrease in accordance with the neck angle at $0^{\circ}-90^{\circ}$ (Cook Zenith Flex device from 59.8 to 48.9 N, Medtronic Endurant device from 29.9 to $25.8 \mathrm{~N}$, and Medtronic Talent device from 6.0 to $5.5 \mathrm{~N}) .{ }^{18)}$ From another point of view, De Bock et al. reported using the in vitro test to show that proximal kinking of the device can occur and result in the presence of type Ia endoleak in an angle between the suprarenal aorta and proximal neck above $60^{\circ}{ }^{14)}$

Similar to previous studies, ${ }^{12-17)}$ the current study revealed that the incidence of persistent type Ia endoleak was higher in the angulated group, while its freedom rate for persistent type Ia endoleak was found to be significantly lower $(\mathrm{p}=0.010)$. This fact indicated that countermeasures against severe proximal neck angulation during EVAR should be mandatory. As other special technique for proximal neck angulation, snorkel technique has also been reported to prevent the occurrence of type Ia endoleaks. ${ }^{19,20)}$ In the future, this method may be considered for long-term treatment in addition to current strategies.

In terms of the mechanism of persistent type Ia endoleak due to proximal neck angulation, the pull-down forces 
by angulation for each device might be considered ${ }^{18)}$ as the different HR of each devices led us to speculate the influence of different trackabilities. However, this speculation should be investigated through a randomized controlled trial (to avoid the selection bias of the device) to ensure the relation between the persistent type Ia endoleak and the specific devices. Additionally, our multivariable analysis indicated the significant effect of reverse-tapered neck for persistent type Ia endoleak. ${ }^{21)}$ Therefore, the additional complex factors including the different trackabilities of a device and reverse-tapered shape of the angle of the proximal LZ were suggested as the mechanism of persistent type Ia endoleak post-EVAR. However, the number of patients having reverse-tapered neck was only $8(5.0 \%)$, and this was not identified as a predictor of sac growth post-EVAR; thus further investigation is needed to resolve this issue.

\section{Limitations}

One of the limitations of our study is having a small sample size for observational retrospective study on a specific cohort who underwent EVAR. Moreover, several data were excluded from the study due to an isolated iliac aneurysm, additional embolization procedure, usage of warfarin, or an insufficient follow-up survey. There was no major device identified as the risk factor for sac enlargement post-EVAR. These results were not presented in this study, because no randomized study was conducted and aneurismal features including proximal neck conditions were not matched. Lastly, the effects of postoperative type II endoleak on sac enlargement could not be completely denied, even if it was statistically not significant.

\section{Conclusion}

The angle of the proximal LZ was found to be an independent risk factor for the development of aneurysm sac growth post-EVAR. The incidence of persistent type Ia endoleaks was found to be significantly higher in the angulated group. Patients with a neck angulation of $47^{\circ}$ or more could be considered as the high-risk group for sac growth post-EVAR.

\section{Disclosure Statement}

All authors have no conflict of interest.

\section{Author Contributions}

Data collection: YS, HM

Writing: YS, HM

Critical review and revision: all authors

Final approval of the article: all authors
Accountability for all aspects of the work: all authors

\section{Supplementary Materials}

Supplementary materials are available at the online article sites on J-STAGE and PMC.

\section{References}

1) EVAR trial participants. Endovascular aneurysm repair versus open repair in patients with abdominal aortic aneurysm (EVAR trial 1): randomised controlled trial. Lancet 2005; 365: 2179-86.

2) EVAR trial participants. Endovascular aneurysm repair and outcome in patients unfit for open repair of abdominal aortic aneurysm (EVAR trial 2): randomised controlled trial. Lancet 2005 ; 365 : 2187-92.

3) Lederle FA, Freischlag JA, Kyriakides TC, et al. Outcomes following endovascular vs open repair of abdominal aortic aneurysm: a randomized trial. JAMA 2009; 302: 1535-42.

4) Schanzer A, Greenberg RK, Hevelone N, et al. Predictors of abdominal aortic aneurysm sac enlargement after endovascular repair. Circulation 2011; 123: 2848-55.

5) Hwang D, Park S, Kim HK, et al. Reintervention rate after open surgery and endovascular repair for non ruptured abdominal aortic aneurysms. Ann Vasc Surg 2017; 43: 134-43.

6) Beckerman WE, Tadros RO, Faries PL, et al. No major difference in outcomes for endovascular aneurysm repair stent grafts placed outside of instructions for use. J Vasc Surg 2016; 64: 63-74.e2.

7) Seike Y, Tanaka H, Fukuda T, et al. Influence of warfarin therapy on the occurrence of postoperative endoleaks and aneurysm sac enlargement after endovascular abdominal aortic aneurysm repair. Interact Cardiovasc Thorac Surg 2017; $24: 615-8$.

8) Lederle FA, Wilson SE, Johnson JR, et al. Variability in measurement of abdominal aortic aneurysms. J Vasc Surg 1995; 21: 945-52.

9) Hobo R, Kievit J, Leurs LJ, et al. Influence of severe infrarenal aortic neck angulation on complications at the proximal neck following endovascular AAA repair: a EUROSTAR study. J Endovasc Ther 2007; 14: 1-11.

10) Walker J, Tucker LY, Goodney P, et al. Adherence to endovascular aortic aneurysm repair device instructions for use guidelines has no impact on outcomes. J Vasc Surg 2015; 61: 1151-9.

11) Lee JT, Ullery BW, Zarins CK, et al. EVAR deployment in anatomically challenging necks outside the IFU. Eur J Vasc Endovasc Surg 2013; 46: 65-73.

12) AbuRahma AF, Campbell JE, Mousa AY, et al. Clinical outcomes for hostile versus favorable aortic neck anatomy in endovascular aortic aneurysm repair using modular devices. J Vasc Surg 2011; 54: 13-21.

13) AbuRahma AF, Yacoub M, Mousa AY, et al. Aortic neck anatomic features and predictors of outcomes in endovascular repair of abdominal aortic aneurysms following vs not following instructions for use. J Am Coll Surg 2016; 222: 579-89.

14) De Bock S, Iannaccone F, De Beule $M$, et al. What if you 
stretch the IFU? A mechanical insight into stent graft Instructions For Use in angulated proximal aneurysm necks. Med Eng Phys 2014; 36: 1567-76.

15) Hoshina K, Ishimaru S, Sasabuchi Y, et al. Japan Committee for Stent graft Management (JACSM)*. Outcomes of endovascular repair for abdominal aortic aneurysms: a nationwide survey in Japan. Ann Surg 2019; 269: 564-73.

16) Oliveira NFG, Gonçalves FB, Hoeks SE, et al. Long-term outcomes of standard endovascular aneurysm repair in patients with severe neck angulation. J Vasc Surg 2018; 68: 1725-35.

17) Malas MB, Hicks CW, Jordan WD Jr, et al. Five-year outcomes of the PYTHAGORAS U.S. clinical trial of the Aorfix endograft for endovascular aneurysm repair in patients with highly angulated aortic necks. J Vasc Surg 2017; 65: 1598607.

18) Rahmani S, Grewal IS, Nabovati A, et al. Increasing angula- tion decreases measured aortic stent graft pullout forces. J Vasc Surg 2016; 63: 493-9.

19) Raux M, Patel VI, Cochennec F, et al. A propensity-matched comparison of outcomes for fenestrated endovascular aneurysm repair and open surgical repair of complex abdominal aortic aneurysms. J Vasc Surg 2014; 60: 858-63; discussion, 863-4.

20) Quatromoni JG, Orlova K, Foley PJ 3rd. Advanced endovascular approaches in the management of challenging proximal aortic neck anatomy: traditional endografts and the snorkel technique. Semin Intervent Radiol 2015; 32: 289-303.

21) Pitoulias GA, Valdivia AR, Hahtapornsawan S, et al. Conical neck is strongly associated with proximal failure in standard endovascular aneurysm repair. J Vasc Surg 2017; 66: 168695. 